\title{
BIOMOLECULAR SENSING USING SURFACE MICROMACHINED SILICON PLATES
}

\author{
A.M. Zapata ${ }^{1}$, E.T. Carlen ${ }^{2}$, E.S. Kim ${ }^{1}$, J. Hsiao ${ }^{1}$, D. Traviglia ${ }^{1}$, M.S. Weinberg ${ }^{1}$ \\ ${ }^{1}$ The Charles Stark Draper Laboratory, Cambridge, MA, USA \\ (Tel : 617-258-1114; E-mail: azapata@draper.com) \\ ${ }^{2}$ The University of Twente and MESA+ Institute for Nanotechnology, Enschede, The Netherlands \\ (Tel : +31-53-489-2661; E-mail: e.t.carlen@ewi.utwente.nl)
}

\begin{abstract}
Micromachined sensors to detect surface stress changes associated with interactions between an immobilized chemically selective receptor and a target analyte are presented. The top isolated sensing surface of a free-standing silicon plate is prepared with a thin Au layer, followed by covalent attachment of chemical or biomolecule forming a chemically-selective surface. Surface stress changes in air are measured capacitively due to the formation of an alkanethiol self-assembled monolayer (SAM). Detection of biomolecular binding in liquid samples is measured optically using the streptavidin-biotin complex and a $M$. tuberculosis antigen-antibody system used for clinical tuberculosis (TB) diagnosis.
\end{abstract}

Keywords: Surface stress sensors, micromechanical biosensors

\section{INTRODUCTION}

Advances in MEMS have facilitated the development of novel transducers for biochemical sensing that rely heavily on mechanical energy [1]. These types of sensors, including resonant and static platforms such as quartz crystal microbalances and microcantilever beams enable label-free assays that are faster, cheaper and less cumbersome than conventional labeled methods [2]. The surface stress sensors presented here are microfabricated from thin layers of single crystal silicon. The thin layer is suspended with all edges clamped to a silicon substrate; therefore, physically isolating the two plate surfaces. One surface is used for sensing and interfaces directly with the sample solution and the other surface is used for displacement detection, transducing the sensing surface's response to chemical stimuli. This sensor offers a number of advantages compared to other label-free, mechanical platforms: 1) static detection resolution is not affected by quality (Q) factor damping in liquid samples, 2) plate structures are more rigid than cantilever beams and can be easily functionalized and probed using commercially available printing techniques, and 3) unlike cantilever beams, the detection surface is physically isolated from the sensing surface and can be easily integrated with differential capacitance read-out electronics and microfluidic handling systems for liquid sensing applications [3]. The surface stress sensing mechanism is different than resonant mass sensing, where the resolution of the resonant mass sensors is typically reduced in a liquid medium due to the reduction of the resonator Q-factor caused by the liquid. Techniques have been developed to improve this problem [4], however, with increased sensor complexity. Surface stress sensors detect low frequency deflection changes of mechanical structures due to differential surface stress changes of a sensing surface. Therefore, the resolution of the surface stress sensors is minimally affected by viscous damping. Surface stress sensors are affected in aqueous environments include by the pressure head of the sample solution, surface tension of the sample solution and ionic strength of the sample solution.

\section{THEORY AND DESIGN}

Surface stress has been previously described mathematically as $\sigma_{\mathrm{ij}}=\delta_{\mathrm{ij} \gamma}+\delta \gamma / \delta \varepsilon_{\mathrm{ij}}$ [5], where the tensors can be represented as scalars for surfaces with lattice symmetry of 3-fold or larger, $\sigma_{\mathrm{s}}$ $\left(\mathrm{Nm}^{-1}\right)$ is the surface stress, $\left(\mathrm{Jm}^{-2}\right)$ is the surface free energy, and $\varepsilon$ the strain. The concept of surface stress implies that the surface stress performs work when straining a solid structure. In thin samples, surface stress can produce measurable elastic bending and deflections.

Small plate deflections due to a uniform axial surface stress are used: (a) the plate material is homogeneous with uniform thickness $t$, (b) $t<$ $b / 10$, where $b$ is the smallest plate dimension, and (c) the maximum deflection $w_{\mathrm{m}}<t / 2[6]$, and (d) 
large deflection shearing forces and body forces are not considered. Fig. 1 shows plate details.

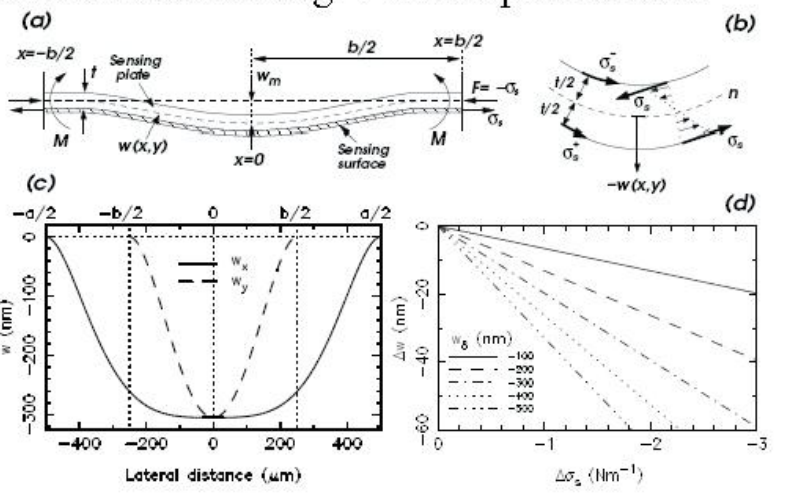

Fig. 1 (a) Plate dimensions and forces: width $b$, thickness $t$, and $\sigma_{s}$ (shown compressive) (b) Bending due to $\sigma_{s}(c)$ Rectangular plate bending profiles for $a=2 b$ and $w_{s}=305 \mathrm{~mm}$ (d) Center deflection $\Delta w$.

Assuming uniform axial stress on the plate surface $\sigma_{s}^{+} \neq \sigma_{s}^{-}$, a differential surface stress $\sigma_{\mathrm{s}}=\sigma_{\mathrm{s}}^{+}$ $\delta(z-t / 2)-\sigma_{\mathrm{s}}^{-} \delta(z+t / 2)$, where $\delta$ is the Dirac-delta function, generates a stress couple of radial flexure moment $M$, shown in Fig. 1(b). This is equivalent to a force $F$ at the neutral surface $n$ such that the resultant force and moment on the edge are equal to zero. This approximation is valid near the plate center $(x=0)$ where all deflections are measured. The total plate deflection $w_{m}$ consists of two terms: one term due to an initial deflection $w_{\delta}$ and an additional deflection $\Delta w$ due to a radial surface force induced by the adsorption of the target molecule on the sensing surface. In practice, it is rare that suspended silicon plates are perfectly flat. All suspended plates presented here have initial plate bending due primarily to the residual stress in the nucleation layer [3]. Since $w_{\delta}$ is much larger than $\Delta w$, then $w_{\delta}$ must be considered when calculating $\Delta \sigma_{\mathrm{s}}$. The total plate bending can be determined by the deflection produced by the combination of a uniformly distributed lateral force $q\left(\mathrm{Nm}^{-2}\right)$, which is related to $w_{\delta}$, and a uniform in-plane force $\mathrm{F}\left(\mathrm{Nm}^{-1}\right)$, which is related $\sigma_{s}$. The bending of a rectangular plate with clamped edges is estimated as $w(x, y)=$ $w_{\delta} / \Gamma_{0}\left(1+\gamma \sigma_{s}\right) \Gamma(x, y)[3]$, where $\gamma$ is an estimated constant, $\Gamma(x, y)$ is a shape function, $\Gamma_{0} \equiv \Gamma(0,0)$. Fig. 1(c) shows an example of the rectangular plate bending. The differential surface stress change can be estimated as $\Delta \sigma_{s} \approx \Delta w / w_{\delta}$ [3]. Fig. 1(d) shows the dependence of $\Delta \sigma_{\mathrm{s}}$ and $\Delta w$ on $w_{\delta}$.
Although, the nucleation layer covers the entire plate surface in this article, surface stress induced deflections can be increased by partially covering the plate surface, therefore, the bending moment due to the edge of the nucleation layer adds to the total bending moment of the plate.

\section{MICROFABRICATION}

The silicon plates were fabricated using conventional surface micromachining processes with silicon-on-insulator (SOI) substrates and deep reactive ion etching (DRIE). Capacitive sensors were fabricated using seven lithography steps. Fig. 2(a) highlights the essential aspects of the microfabrication process. A $1 \mu \mathrm{m}$-thick oxide (LTO1) etch mask film is deposited on the SOI substrates in a low-pressure chemical vapor deposition (LPCVD) system, followed by lithographical patterning and reactive-ion-etching (RIE) to define the sense plate. A $1.0 \mu \mathrm{m}$ thick low-stress LPCVD nitride anchor layer is deposited, patterned, and RIE, thus defining the upper electrode anchor layer, shown in Fig. 2(a,ii).

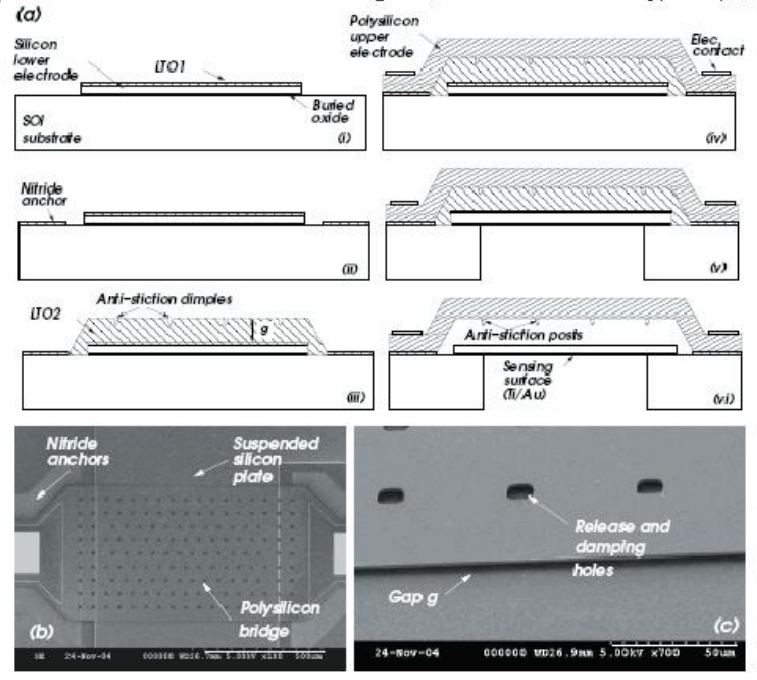

Fig. 2: (a) Simplified sensor microfabricati on process flow (b) Top view SEM of released sensor structure (c) SEM of released capacitor structure.

The remaining LTO1 mask layer is removed in a dilute hydrofluoric acid (HF) solution. A $3 \mu \mathrm{m}$ thick LPCVD oxide layer (LTO2) is deposited defining the separation gap. The LTO2 layer is then patterned and etched as shown in Fig. 2(a,iii). The $4 \mu$ m-thick LPCVD low-stress polysilicon layer is next deposited, patterned and etched (RIE) thus opening the release holes and defining the upper polysilicon plate structure. Electrical 
contacts are formed by metallization $(30 \mathrm{~nm}$ $\mathrm{Cr} / 500 \mathrm{~nm} \mathrm{Au}$ ) and liftoff, shown in Fig. 2(a,iv). Next, the handle layer is patterned and etched using DRIE, shown in Fig. 2(a,v). Next, the handle layer is patterned and etched using DRIE, shown in Fig. 2(a,v). The remaining oxide layers are then removed in a $3: 1 \mathrm{HF}: \mathrm{H}_{2} \mathrm{O}$ solution, shown Fig. 2(a,vi). Sensor structures for optical detection were also fabricated from SOI substrates using two lithography steps to define and release the sensing plate, shown in Figs. 2(a,i) and 2(a,v). Studies of biomolecular interactions in liquid samples are facilitated by fabricating a 3-D silicon -PDMS (polydimethylsiloxane) hybrid structure using simple replica molding and bonding processes [7]. The PDMS structure is made from a master mold from a silicon substrate. A curing agent and PDMS prepolymer (Sylgard 184 Silicone Elastomer, Dow Corning) are mixed in 1:10 weight ratio, and degassed in a vacuum desiccator. The prepolymer mixture is then poured onto the master mold and cured at $70^{\circ} \mathrm{C}$. After curing, the PDMS mold replicas are peeled off from the mold and cut to fit the dimensions of the microsensor die. The final structure is a $2-\mathrm{mm}$ thick square layer containing a v-shaped channel with dimensions of $1.75 \mu \mathrm{m} \times 3.5 \mu \mathrm{m} \times 200 \mu \mathrm{m}$, shown in Fig. 3. Both silicon and PDMS surfaces

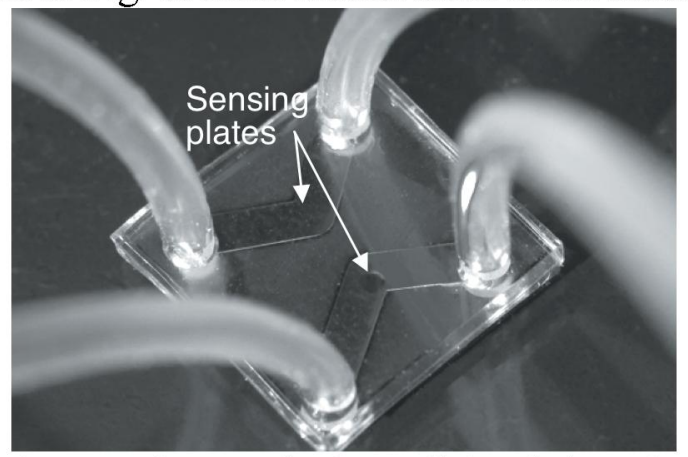

Fig. 3: PDMS microfluidic cell bonded to 2-sense plate device (900 $\mu \mathrm{m}$-diameter), inlet/outlet tubing.

are activated by oxygen plasma (100 mTorr, 100 $\mathrm{W})$ for $30 \mathrm{~s}$. The process generates hydrophilic surfaces that can form a permanent bond. Plasma treated surfaces are immediately aligned and contacted to initiate bonding, and left for $20 \mathrm{~min}$. while applying pressure. Coring of the PDMS and insertion of tygon tubing (Dow Corning, No. 2 tubing) enables the off-chip fluidic connections. The volume of reagent needed to fill the $\mathrm{v}$-shaped cell is $6 \mu \mathrm{L}$.

\section{EXPERIMENTS AND RESULTS}

Self-assembling alkanethiol monolayers on thin $\mathrm{Au}(111)$ layers are used for plate bending characterization in ambient air. The high affinity of thiols for gold surfaces facilitates their use to generate well-defined organic surfaces with a wide range of chemical functionalities displayed at the sensing interface. The bottom side of the silicon plates is sputtered coated with a $30 \mathrm{~nm} \mathrm{Au}$ nucleation layer $(8 \mathrm{~nm}$ Ti adhesion layer). Capacitance measurements are done with custombuilt electronics circuitry, in a low-noise commonmode rejection configuration, shown in Fig. 4(b).

(a)

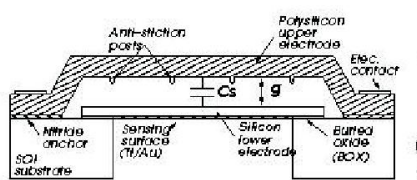

(c)

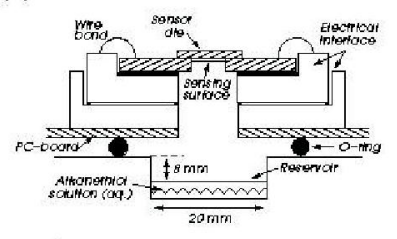

Fig. 4: (a) Sensor cross capacitance measurement circuit (c) test fixture (d) measured 1-dodecanethiol response where $\Delta \sigma_{s}$ plotted with $w_{\delta}=305 \mathrm{~nm}, \mathrm{~g}=3.2 \mu \mathrm{m}$ and $b=480 \mu \mathrm{m}$.

Sense and reference capacitors are driven by a modulation signal, and the differential capacitance is converted to a voltage with a charge-integrating amplifier. Prior to testing, the sensor baseline is first established for $60 \mathrm{~s}$ before exposing the sensing surface to the test vapor. The Au coated sensing surface is then exposed to the alkanethiol vapor from a large sealed reservoir of liquid $\approx 1.3$ $\mathrm{mL}$ for a time of $300 \mathrm{~s}$. Fig. 4(b) shows the sensor response of a test device. Prior to exposure, the offset voltage of $\approx 500 \mathrm{mV}$ is consistent with initial plate deflection of $w_{\mathrm{d}} \approx 305 \mathrm{~nm}$. The exposure of the sensing surface at $\mathrm{t}=60 \mathrm{~s}$ to vapor phase 1-dodecanethiol (Aldrich No. 471364) results in a surface stress change $\Delta \sigma_{\mathrm{s}} \approx 0.42 \mathrm{Nm}^{-1}$, smaller than values previously reported by our group using optical interferometry [3], but similar to other reports [8].

Deflections induced by binding of a biological receptor immobilized at the plate's sense surface 
to an analyte flowing in a liquid matrix, were studied using the optical lever detection method commonly used with atomic force microscopy. An immunological diagnostic model for $M$. tuberculosis based on the binding of the bacterium's Early Secretory Antigen Target 6, (ESAT-6) to its corresponding polyclonal antibody (anti-ESAT-6) was used. The ESAT-6 antigen was immobilized at the Au coated surface using dithiobis-[succinimidylproprionate] (DSP), a thiolated crosslinker for $\mathrm{Au}$ binding, and a primary amine-reactive NHS ester for amide crosslinking to proteins. Following DSP surface activation, the sensor is incubated overnight with ESAT-6, and then placed on the optical lever setup. All reagents are introduced into the PDMS channels manually with $1-\mathrm{ml}$ syringes and 19G ss needles. Fig. 5 shows and example of the sensor response to $120 \mathrm{nM}$ anti-ESAT-6. The data has

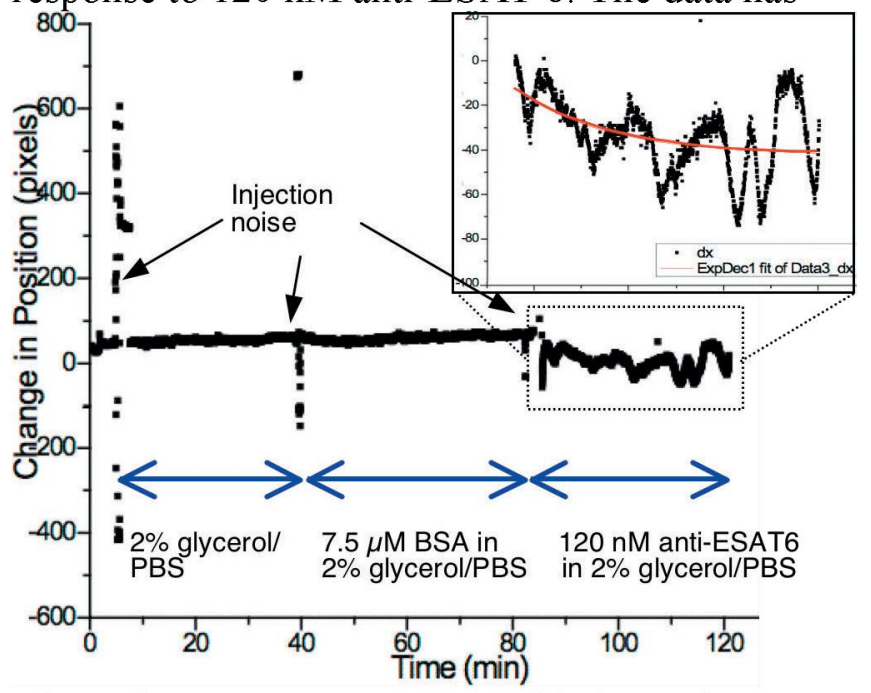

Fig. 5: Sensor response to anti-ESAT6 introduction.

not been normalized for pressure and temperature perturbations, therefore the sample injection spike and temperature relaxation can be seen. The sensor's baseline is first established with PBS containing $2 \%$ glycerol, representative of the antiESAT-6 matrix. No deflections due to nonspecific binding of $7.5 \mu \mathrm{M}$ BSA solution are observed. The response to BSA was essentially the same as observed for the PBS solution. Introduction of $120 \mathrm{nM}$ anti-ESAT- 6 generated a measurable response markedly different, and most likely attributed to antigen-antibody binding. The noise during binding is likely due to bubbles in the PDMS channels and observed in previous experiments. Larger antibody concentrations (not show) yielded larger response magnitudes. Surface plasmon resonance (SPRImagerII, GWC Technologies) studies confirm the association binding rate for the TB system does not follow the expected Langmuir adsorption kinetics $(\mathrm{k} \sim 19000$ $\mathrm{M}^{-1} \mathrm{~s}^{-1}$ ), likely due to non-specific binding.

\section{CONCLUSIONS}

The microfabricated surface stress plate sensors presented here are advantageous, in our view, compared to cantilever beam structures in three important ways: 1) not affected by degradation of quality factor, 2) plate structures are less fragile more than beams and 3 ) the detection surface is physically isolated from the sensing surface and easily adapted to other readout techniques in liquid solutions. Although the microfabrication technology used to manufacture the plate sensors is more complex than that used for cantilever beams, surface micromachining technology is well established and provides a path to low-cost mass production of sensors. The measurements presented here indicate that the plate structures with electronic readout are as sensitive as the cantilever beam-optical readout systems.

\section{ACKNOWLEDGMENTS}

The authors thank Draper Laboratory for funding, Connie Cardoso, Mert Prince, and Manuela Healey for fabrication assistance, John Lachapelle for electronics, Caroline Kondoleon for packaging, and Fusion Antibodies for the tuberculosis immunological reagents.

\section{REFERENCES}

[1] N.V. Lavrik, M.J. Sepaniak, P.G. Datskos, Rev. of Sci. Instruments, vol. 75, No. 7, pp. 2229, 2004

[2] P. Skladal, J. Braz. Chem. Soc., vol.14, No.4, pp. 491, 2003.

[3] E.T. Carlen, M.S. Weinberg, C.E. Dube, A.M. Zapata, J. T. Borenstein, App. Phys. Let., vol.89, pp. 173123, 2006.

[4] T. Burg and S. Manalis Appl. Phys. Lett., vol.83, No.13, pp. 2698,2003

[5] R. Shuttleworth Proc. Phys. Soc.,vol.A63, pp. 444, 1950. [6] S. Timoshenko, Theory of Plates and Shells. McGrawHill Book Company, Inc., 1959

[7] B.H. Jo, L.M. Van Lerberghe, K.M. Motsegood and D. J. Beebe, J. Microelectromech. Sy st.,vol.9, No.1, p. 76, 2000. [8] M. Godin, P. Williams, V. Tabard-Cossa, O. Laroche, L. Beaulieu, R. Lennox, and P. Grütter, Langmuir, vol. 20, p. $7090,2004$. 